\title{
Vibration-proof High-pressure Xenon Electroluminescence Detector
}

\author{
Alexander Bolozdynya and Raymond DeVito
}

\begin{abstract}
We have developed a high-pressure electroluminescence (EL) detector consisting of a cathode, 5 drift rings, and an EL-generating structure which defines a sensitive volume of $\varnothing 5 \mathrm{~cm} \times 5 \mathrm{~cm}$. The EL-generating structure consists of two parallel-plate, chemically etched grids and a high-pressure optical window which is optically coupled to an external photomultiplier tube. Ionizing radiation that is absorbed in the sensitive volume generates electrons, which drift into the EL region and produce an EL flash. The detector was filled with 20bar Xe gas that was highly purified ( $>1$ msec electron-life time) using a spark purification technique. In order to evaluate the potential for using an EL detector under adverse conditions, we disturbed the detector using a $10 \mathrm{~W}$ electric engraver working at $60 \mathrm{~Hz}$. The action of the engraver had practically no effect on spectra acquired from an ${ }^{241} \mathrm{Am}$ source.
\end{abstract}

\section{INTRODUCTION}

In the past decade, significant progress has been achieved in the development of high-pressure xenon (HPXe) ionization chambers (see, for example, [1] and references therein). These detectors have demonstrated that good energy resolution is achievable with kilograms of compressed Xe operating at room temperature. The most common method of acquiring data from HPXe detectors is to measure the charge induced on electrodes by drifting ionization electrons. To eliminate the dependence of the pulse amplitude on interaction position, the ionization chamber is often divided into two parts by a screening Frisch grid [2]. The grid is maintained at an intermediate potential between the cathode and the anode, making it electrically transparent to drifting ionization electrons. The maximum voltage pulse amplitude induced at electrodes from collection of $n_{0}$ electrons is given by $V_{\max }=n_{0}$ $e / C_{D}$, where $C_{D}$ is a capacitance of the grid relative to the anode. So, the ionization signal is very sensitive to the changing capacitance due to micro-vibrations of the grid and the anode. This microphonic effect is the most important factor limiting performance of HPXe detectors

Another method to extract information from high-pressure noble gas ionization chambers is to detect excitation light generated by ionization electrons drifting along the electric field through the gas (see, for example [3] and references therein). In the presence of high enough electric field, ionization electrons can gain sufficient energy between successive collisions to cause excitation of atoms or secondary ionization. If the energy of drifting electrons is slightly below the ionization threshold, they do not initiate chargemultiplication avalanches but rather effectively excite noble gas atoms, $A$, and generate intense electroluminescence light (EL) by the following processes:

$$
\begin{gathered}
e+A \rightarrow e+A^{*} \\
A^{*}+2 A \rightarrow A_{2} *+2 A, \text { at gas densities }>10^{10} \mathrm{~cm}^{-3} \\
A_{2}{ }^{*} \rightarrow 2 A+h \mathrm{v} .
\end{gathered}
$$

For a given gas pressure, the electroluminescence process does not occur below a certain field threshold. In a uniform electric field, the number of photons, $N_{p h}$, generated by one drifting electron is proportional to the drift path $x[\mathrm{~cm}]$, the reduced electric field strength $E / p[\mathrm{kV} / \mathrm{cm} / \mathrm{bar}]$, and the gas pressure $p$ [bar]:

$$
N_{p h}=70(E / p-1.0) p x \text { UV photons/e cm drift; }
$$

The best value of the intensity of electroluminescence has been measured to be 1,700 photons $/ \mathrm{cm}$ at $E / p=3.4 \mathrm{kV} / \mathrm{cm} / \mathrm{bar}$ [4]. EL has been observed in all noble gases and their mixtures. In gas mixtures containing $>0.1 \% \mathrm{Xe}$, the lightoutput and spectrum of electroluminescence is very similar to that of pure xenon. The EL process is not something specific only for noble gases. The effect is widely used in solid semiconductor devices such as LEDs [5]. Most importantly, as one can see from equation (1), the electroluminescence signal is not sensitive to the capacitance of the electrode system, and in a uniform electric field, the signal is proportional to the voltage drop between electrodes rather than the electric field strength. This means that the principal factor limiting the performance of ionization chambers can be eliminated in electroluminescence detectors. The process of electroluminescence is linear, in contrast to the exponential behavior of electron multiplication. Due to this circumstance, EL benefits from lower fluctuations and better energy resolution than the gas gain amplification process. Advantages of EL detectors in getting good energy resolution especially at 9-20 bar pressures have been already demonstrated $[3,4]$. The goal of this work is to show that ELDs are relatively insensitive to vibrations.

\section{METHODS}

A schematic design of the EL detector used in this study is presented in Fig.1. The cathode (2), drift electrodes (3), and the grid (4) define a sensitive volume having dimensions of $\varnothing 5 \mathrm{~cm} \times 5 \mathrm{~cm}$. Parallel-plate photolithography-made grid electrodes $(4,5)$ are used to generate electroluminescence that is detected by a photomultiplier (9) optically coupled to a window placed behind the grounded grid. A $0.5 \mathrm{mg} / \mathrm{cm}^{2}$ layer of p-terphenyl $\left(\mathrm{C}_{14} \mathrm{H}_{18}\right)$, serving as a wave shifter, is vacuum-deposited on the input surface of the optical window enabling the photomultiplier to "see" the 170-nm UV light generated during the electroluminescence process. The quantum efficiency of the p-terphenyl wave shifter has been 
measured to be $>90 \%$ in pressurized xenon [4]. The emission spectrum of p-terphenyl has 2 peaks: one at 350 and another at $450 \mathrm{~nm}$. An important property of this well-known scintillating dye is that it doesn't contaminate xenon. Highvoltage feedthroughs (8) are installed in the flange (7) to supply voltage to the electrodes. The flange is equipped with a knife-edge groove for 2-3/4" CF gaskets, which were used for installation of 10 -mm thick UV-grade optical window (KU-8). A blue-sensitive EMI THORN 9125B photomultiplier with 30 -mm diameter input window was optically coupled to the external face of the window.

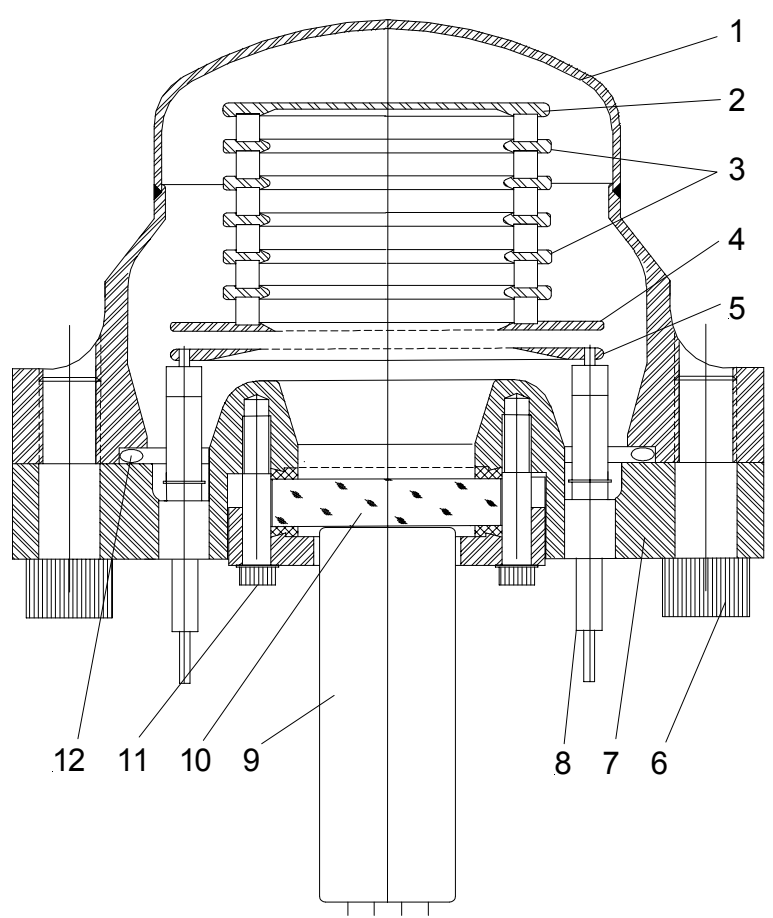

Fig.1. Electroluminescence high-pressure xenon detector with parallel-plate electrode system and photomultiplier readout: 1- high-pressure vessel, 2 aluminum cathode, 3 - drift electrodes separated by stand-off ceramic insulators, 4 and 5 grid electrodes forming electroluminescence region, 6 bolt, 7 - flange, 8 - HV feedthroughs, 9 - photomupltiplier, 10 - optical window, 11 - bolt, 12 - Helocoflex gasket in aluminum jacket. Sensitive volume of the detector enclosed into the electrode system of 2-4 has 5-cm diameter and 5-cm depth.

Before assembly, all metal and ceramic-made detector parts were baked at $200^{\circ} \mathrm{C}$ under a vacuum of $<10^{-6}$ Torr. The assembled detector was pumped down to $10^{-8}$ Torr for a week before filling with xenon. We used pure $\mathrm{Xe}$ or $\mathrm{Xe}+0.2 \% \mathrm{H}_{2}$ gas mixture to fill the detector. A spark purification technique has been used to remove electronegative impurities from the gas. The ultimate purity of used gases corresponded to several milliseconds of electron lifetime.

In this project we have used standard NIM electronics and a desktop computer with an APTEC-NRC 5016 MCA card providing data acquisition, display, peak search and analysis. A high-voltage potential of up to $30 \mathrm{kV}$, filtered with a custom made HV filter, was applied to the anode of the detector. A cathode bias of up to $20 \mathrm{kV}$ was supplied using a separate $\mathrm{HV}$ power supply. The system includes a LeCroy LT346L Waverunner digital scope for recording and pulse shape analysis.

The detector operates in the following manner. Ionization radiation absorbed in the sensitive volume generates electrons, which drift into the EL region and generate an EL flash. UV light is shifted into the $350-450 \mathrm{~nm}$ range by p-terphenyl deposited on the inside surface of the window. The PMT detects the $350-450 \mathrm{~nm}$ photons.

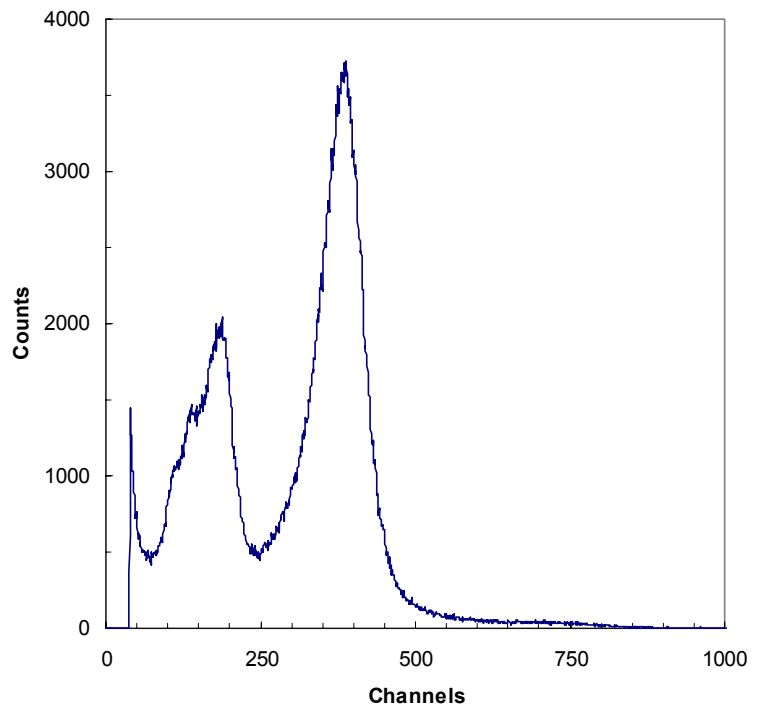

Fig.2. Spectrum of ${ }^{241} \mathrm{Am}$ measured in $20 \mathrm{bar} \mathrm{Xe}$

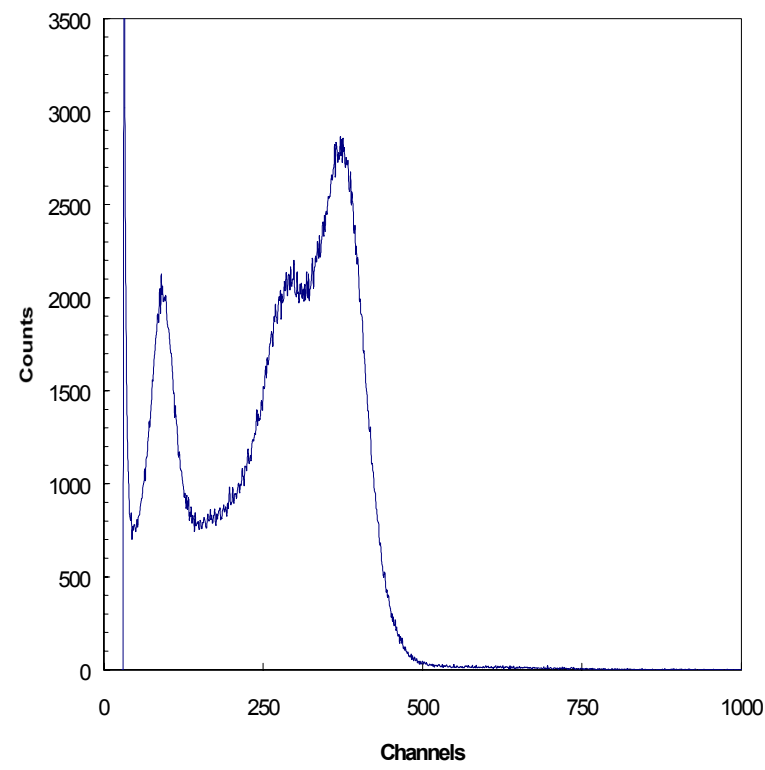

Fig.3. Spectrum of ${ }^{57} \mathrm{Co}$ measured in 21 bar $\mathrm{Xe}+0.2 \% \mathrm{H}_{2}$ 


\section{RESULTS}

The EL detector was tested with pure $\mathrm{Xe}$ and a $\mathrm{Xe}+0.2 \% \mathrm{H}_{2}$ gas mixture pressurized up to 31 bar. The best energy resolution has been achieved at pressures of about 20 bar. In Fig.2, there is shown a pulse height spectrum obtained using a ${ }^{241}$ Am gamma source installed in the center of the cathode (2, Fig.1). The highest peak represents photoabsorption of 59.6 $\mathrm{keV}$ gamma rays in the sensitive volume of the detector. The next peak on the left is a superposition of the escape peak and photoabsorption of fluorescence photons $(29.7 \mathrm{keV}$ and 33.8 keV). In Fig.3, there is shown a pulse height spectrum measured with ${ }^{57} \mathrm{Co}$ gamma source placed outside the detector. The highest double peak represents photoabsorption of 122 $\mathrm{keV}$ gamma rays and escape peak $(\sim 92 \mathrm{keV})$. The next peak on the left represents photoabsorption of the fluorescence photons.

In order to prove the statement about vibration insensitivity of the ELD, we used an electric engraver $(10 \mathrm{~W}, 60 \mathrm{~Hz})$ to disturb the ELD. The writing pin of the engraver was installed in the flange of the ELD. Pulse height spectra of an ${ }^{241} \mathrm{Am}$ gamma source were taken for the same acquisition time while the engraver was working and when it was turned off. No significant difference between spectra in the range of $>10 \mathrm{keV}$ was found (Fig.4). We tried to repeat the test with a few Constellation HPXe ionization chambers [6] and found that the working engraver generated enormous signals exceeding the ionization signals from the gamma sources by two orders of magnitude. The microphonic effect prohibits any spectral measurements under these conditions and introduces the risk of damage to the preamplifiers.

\section{CONCLUSION AND DISCUSSION}

The experiment confirmed that vibrations do not affect the performance of an ELD. This property is unique among HPXe detectors and allows the consideration of ELDs for use under very challenging conditions. We plan to improve performance of the ELD by replacing the relatively fragile optical window and PMT with a large avalanche photodiode.

The limiting factor of the ELD is that it requires extremely high voltages for generation of EL signals at high xenon densities. Technically, an ELD is most suitable for work below 30-bar pressure or $0.2 \mathrm{~g} / \mathrm{cm}^{3}$ xenon density, limiting their application for detection of relatively soft gamma radiation. We believe that EL detectors may find widespread use in oil-well logging and in nuclear material monitoring systems, in particular field-deployable systems such aerial or automobile platforms targeted at detecting and identifying special nuclear materials such as weapons grade plutonium.

\section{ACKNOWLEDGMENTS}

The authors gratefully acknowledge the financial support of the SMDC DARPA office. The authors thank Dr. R. Austin for reading and editing the manuscript and Ed Knighton, Scott McPherson, and Leo Godbee for help in development of the instrumentation.

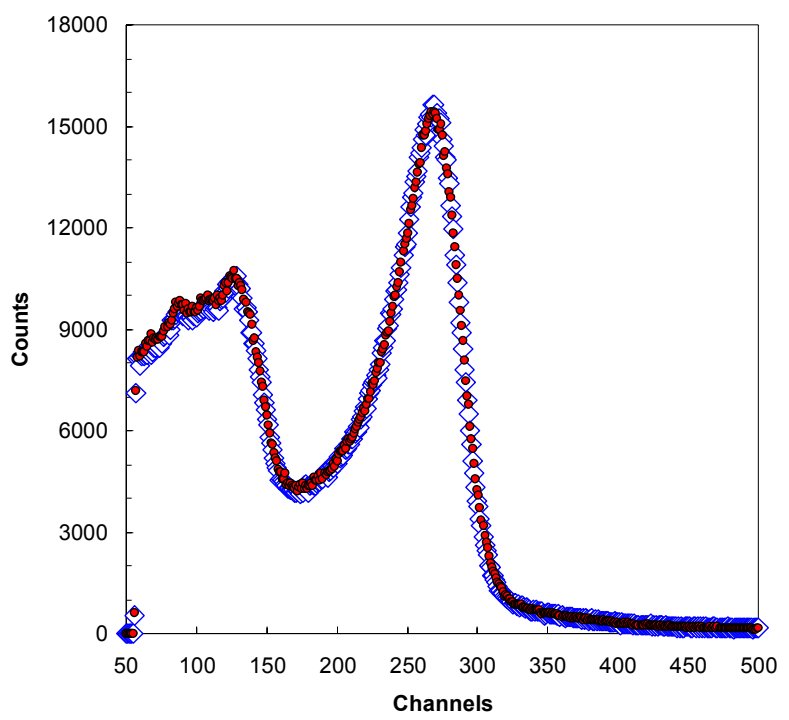

Fig.4. Spectra of ${ }^{241} \mathrm{Am}$ gamma ray source measured in absence (open diamonds) and presence (closed circles) of mechanical vibrations generated by an electric engraver. The detector is filled with 20 bar Xe.

\section{REFERENCES}

[1] A.Bolotnikov and B. Ramsey, "Improving the Energy Resolution of Highpressure Xe Cylindrical Ionization Chambers”, IEEE Trans. Nucl. Sci. 44 (1997) 1006-1010

[2] G.F. Knoll, Radiation Detection and Measurements, $3^{\text {rd }}$ edition, John Wiley \& Sons, 2000.

[3] A.Bolozdynya et. al.,"A high pressure xenon self-triggered scintillation drift chamber with 3D sensitivity in the range of 20-140 keV deposited energy", Nucl. Instr. Meth. A ,v.385 (1997) 225-238.

[4] S. Belogurov e.a., "High pressure Gas Scintillation Drift Chamber with Photomultipliers Inside of Working Medium”, 1995 IEEE Nucl. Sci. Symp. and Medical Imaging Conf. Conference Record, October 21-28, 1995, San Francisco, vol.1, pp.519-523

[5] Electroluminescence I. Semiconductors and Semimetals, Vo.64, Ed. G. Mueller, Academic Press, 2000

[6] A.Bolozdynya, A. Arodzero, and R. DeVito, "High-pressure xenon detectors for applications in portal safeguard systems and for monitoring nuclear waste", INMM $43^{\text {rd }}$ Annual Meeting, Orlando, June 23-27, 2002 